\title{
Selection of UPFC Suitable Locations for System Security Improvement Under Normal and Network Contingencies
}

\author{
K. Visakha \\ D: Thukaram * Lawrence Jenkins \\ Department of Electrical Engineering, \\ Indian Institute of Science, \\ Bangalore - 560012 , INDIA. \\ *Email: dtram@ee.iisc.ernet.in
}

H.P.Khincha

\begin{abstract}
Electric power systems are exposed to various contingencies. Network contingencies often contribute to over-loading of network brànches, unsatisfactory voltages and also leading to problems of stability/voltage collapse. To maintain security of the systems, it is desirable to estimate the effect of contingencies and plan suitable measures to. improve system security/stability. This paper presents an approach for selection of UPFC suitable locations considering normal and network contingencies after evaluating the degree of severity of the contingencies. The ranking is evaluated using composite criteria based fuzzy logic for eliminating masking effect. The selection of UPFC suitable locations uses the criteria on the basis of improved system security/stability. The proposed approach for selection of UPFC suitable locations has been tested under simulated conditions on a few sample power systems and the results for a real life 36-node equivalent EHV power network are presented for illustration purposes.
\end{abstract}

\section{INTRODUCTION}

In power system planning various types of studies are carried out, considering various system operational scenarios. Intended and unintended switching operations are considered for very fast transient conditions and protective measures are planned for the purposes. Under dynamic conditions such as faults, line openings, generator trippings and load throw off etc. protective systems are designed with more emphasis on protecting the equipments than concern to the system security and stability. However, judicious use of dynamic controls at generating systems, excitation/governor systems, HVDC systems, static VAR compensators (SVCs) and more recently FACTS devices will help to maintain the system security/stability. In a day-to-day operation it may be beyond the operators scope to take any control decision during emergencies. However, the operator can use various control devices and also SVCs, UPFC to restore the system to normal conditions. In planning study it may be prohibitive to 'carry' out dynamic studies for an exhaustive contingencies. Thus it is important to evaluate exhaustive numbers of static contingency studies and obtain the set of important severe contingencies for detailed dynamic analysis.
Contingency screening and ranking is one of the important components of on-line system security assessment. Most of the contingency ranking methods, generally, ranks the contingencies in an approximate order of severity with respect to a scalar performance index (PI), which quantifies the system stress [1]-[4]. It has been pointed out that two separate ranking lists are required for real power flow problems and voltage profile problems, respectively, since the contingencies causing line overloads do not necessarily cause bus voltage violations and vice versa. Thus, two performance indices, which give measures for line overloads and for bus voltage violations respectively, are needed for real power and voltage contingency rankings [5]. The common disadvantage of several PI-based ranking methods is the masking phenomenon.

With increased loading of existing power transmission systems, the problem of voltage stability and voltage collapse, has also become a major concern in power system planning and operation. It has been observed that voltage magnitudes do not give a good indicator of proximity to a voltage stability limit and voltage collapse [6,7]. Therefore, in the network contingency ranking, it is necessary to consider voltage stability indices at all the load buses as the post-contingent quantities, in addition to real power loadings and bus voltage violations for estimating the actual system stress under a contingency. Then suitable measures/preventive control actions can be planned to improve system security/stability.

The most comprehensive device emanated from the FACTS initiative is the Unified Power Flow Controller (UPFC) $[8,9]$. The UPFC regulates the active and reactive power control as well as adaptive to voltage magnitude control simultaneously or any combination of them. Controlling the power flows in the network, under normal and network contingencies, help to reduce flows in heavily loaded lines, reduce system power loss, improve stability and performance of the system without generation rescheduling or topological changes [10]. Because of the considerable costs of the FACTS devices, it is important to ascertain the location for placement of these devices suitable for various network contingencies. 


\section{TENCON $2003 / 756$}

The locations of the UPFC device in the power system are obtained on the basis of static and/or dynamic performances. There are several methods for finding locations of UPFC in vertically integrated systems but little attention has been devoted to power systems under network contingency. In this paper, for selection of suitable locations of UPFC, voltage stability L-index of load buses has been used as the basis for improved system security/stability after evaluating the degree of severity of the considered contingency. The ranking is evaluated using composite criteria based fuzzy approach for eliminating masking effect. The fuzzy approach uses voltage stability indices at the load buses as the post-contingent quantities, in addition to real power loadings and bus voltage violations to evaluate the network contingency ranking. The proposed approach has been tested under simulated conditions on a few power systems and the results for a 24-node real-life equivalent EHV power network are presented for illustration purposes.

$\because$

\section{VOLTAGE STABILITY INDEX (L-INDEX) COMPUTATION}

Consider a system where $\mathrm{n}$ is the total number of buses with $1,2 \ldots \mathrm{g}, \mathrm{g}$ number of generator buses, and $\mathrm{g}+1 \ldots \mathrm{n}$, remaining ( $\mathrm{n}-\mathrm{g})$ buses. For a given system operating condition, using the load flow (state estimation) results, the voltage stability L-index is computed as [6],

$$
\mathrm{L}_{\mathrm{j}}=\left|1-\sum_{i=1}^{g} F_{j i} \frac{V_{i}}{V_{j}}\right|
$$

Where $\mathrm{j}=\mathrm{g}+1 \ldots \mathrm{n}$ and all the terms within the sigma on the RHS of equation (I) are complex quantities. The values of

$\boldsymbol{F}_{j i}$ are obtained from the network $\boldsymbol{Y}$-bus matrix. For a given operating condition

$$
\left[\begin{array}{c}
I_{G} \\
I_{L}
\end{array}\right]=\left[\begin{array}{ll}
Y_{G G} & Y_{G L} \\
Y_{L G} & Y_{L L}
\end{array}\right]\left[\begin{array}{c}
V_{G} \\
V_{L}
\end{array}\right]
$$

Where $I_{G}, I_{L}$ and $V_{G}, V_{L}$ represent complex current and voltage vectors at the generator nodes and load nodes.

$\left[Y_{G G}\right],\left[Y_{G L}\right],\left[\boldsymbol{Y}_{L L}\right]$ and $\left[\boldsymbol{Y}_{L G}\right]$ are corresponding partitioned portions of network $\boldsymbol{Y}$-bus matrix. Rearranging the above equation (2) we get,

$$
\left[\begin{array}{c}
V_{L} \\
I_{G}
\end{array}\right]=\left[\begin{array}{ll}
Z_{L L} & F_{L G} \\
K_{G L} & Y_{G G}
\end{array}\right]\left[\begin{array}{l}
I_{L} \\
V_{G}
\end{array}\right]
$$

Where $\left[F_{L G}\right]=-\left[Y_{L L}\right]^{-1}\left[Y_{L G}\right], F_{j i}$ are the complex elements of $\left[F_{L G}\right]$ matrix.
An $\mathrm{L}$-index value away from 1 and close to 0 indicates an improved system security. For an unloaded system with generator /load buses voltages $1.0 \angle 0$, the $\mathrm{L}$ - indices for load buses are close to 0 (zero), indicating that the system has maximum stability margin. For a given network as the load/generation increases the voltage magnitude and angles change and for near maximum power transfer condition the voltage stability index $L_{j}$ values for load buses tend to close to 1 (unity), indicating that the system is close to voltage collapse. While the different methods give a general picture of the proximity of the system voltage collapse, the L- index gives a scalar number to each load bus. Among the various indices for voltage stability and voltage collapse prediction, the L-index gives fairly consistent results. The advantage of this method lies in the simplicity of the numerical calculation and expressiveness of the results.

\section{THE APPROACH}

The major steps involved in the approach are as follows.

Step 1. For a given system, exhaustive contingency analyses are carried out.

Step 2. Contingency ranking using three criteria viz, line loading, bus voltage profiles and a voltage stability index of load buses is carried out.

Step 3. A contingency ranking method using all the above criterion has been used which employed fuzzy approach. This is described in section 4

Step 4. Since most of the contingencies may not pose system security/stability problems those of contingencies that pose serious system security/stability problems are selected.

Step 5. A set of most severe contingencies, in the order of severity, is identified which needs additional supporting devices such as UPFE to improve the system conditions.

Step 6. Based on the above set of network contingencies, a few transmission corridors are considered for placement of UPFC devices.

Step 7. For each contingency, analyses are carried out with placement of UPFC in different transmission corridors.

Step 8. Based on the improved performance (voltage stability indices) the most suitable location and the next most suitable for UPFC is suggested.

Step 9. From the analysis of all above set of contingencies one or two best locations for UPFC placement are suggested. 


\section{Power Transmission and Distribution / 757}

\section{FUZZY APPROACH FOR CONTINGENCY RANKING}

The post-contingent quantities are first expressed in fuzzy set notation before they can be processed by the fuzzy rules.

\section{Line Loadings}

Each post-contingent percentage line loading is divided into four categories using fuzzy set notations: Lightly Loaded (LL), $0-50 \%$, Normally Loaded (NL), $50-85 \%$, Fully loaded (FL), $85-100 \%$, Over Loaded (OL), above $100 \%$. Fig. 1 shows the correspondence between line loading and the four linguistic variables.

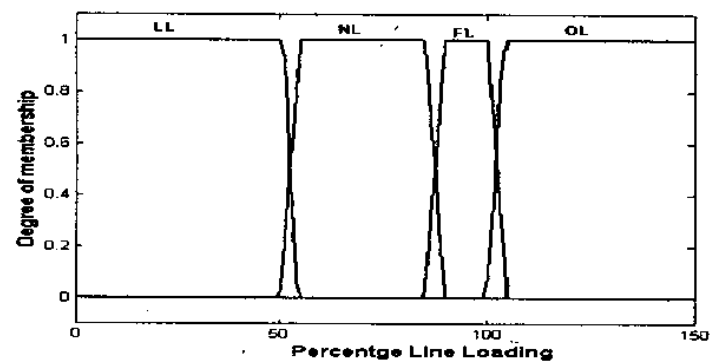

Fig.1 Line Loadings and the corresponding linguistic variables

\section{Bus Voltage Profiles}

Each post-contingent bus voltage profile is divided three categories using fuzzy set notations: Low Voltage (LV), below 0.9pu, Normal Voltage (NV), 0.9-1.02pu and Over Voltage (OV), above 1.02pu. Fig. 2 shows. the correspondence between bus voltage profiles and the three linguistic variables.

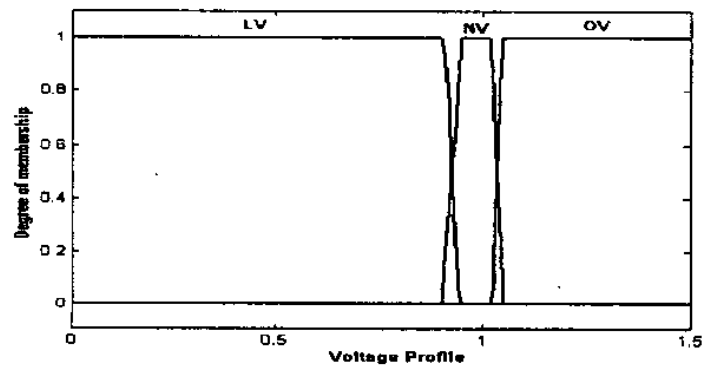

Fig.2 Voltage Profiles and the corresponding linguistic variables

\section{Voltage Stability Indices}

Each post-contingent voltage stability index is divided into five categories using fuzzy set notations: Very Low Index (VLI), 0-0.2, Low Index (LI), 0.2-0.4, Medium Index (MI), 0.4-0.6, High Index (HI), 0.6-0.8and Very High Index (VHI), above 0.8 . Fig. 3 shows the correspondence between bus voitage stability indices and the five linguistic variables.

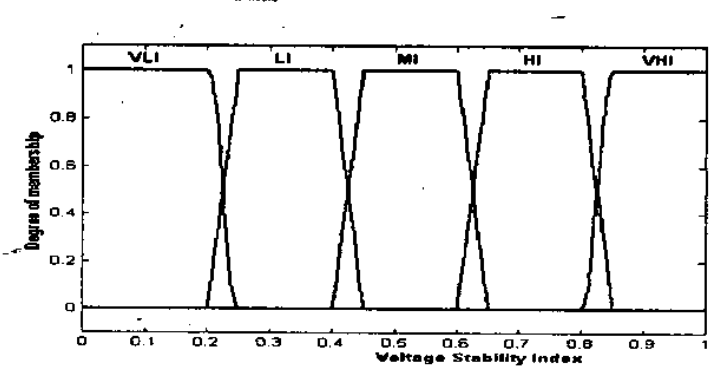

Fig.3 Voltage Stability Index and the corresponding linguistic variables

The fuzzy rules, which are used for evaluation of severity indices (SI) of post -contingent quantities, are given in Table 1.

Table 1

Fuzzy Rules

\begin{tabular}{|lllll|lllll|}
\hline \multicolumn{3}{|c|}{ Post-Contingent Quantity } & \multicolumn{5}{c|}{ Severity } \\
\hline LL & NL & FL & OL & LS & BS & AS & MS \\
\hline LV & NV & OV & & & MS & BS & MS & & \\
\hline VLI & LI & MI & HI & VHI & VLS & LS & BS & AS & MS \\
\hline
\end{tabular}

Where VLS=Very Less Severe, LS $\cdot=$ Less Severe BS=Below Severe, $\quad$ AS= Above Severe . MS=More Severe

The Overall Severity Indices (OSI) for a particular line outage are obtained using the following expressions

$$
\begin{array}{ll}
O S I_{L L}=\sum w_{L L} S I_{L L} & , O S I_{V P}=\sum w_{V P} S I_{V P} \\
O S I_{V S I}=\sum w_{V S I} S I_{V S I} &
\end{array}
$$

Where

$w_{L L}, w_{V P}, w_{V S I}=$ Weighting coefficients for severity indices of line loading, voltage profile and voltage stability indices respectively.

$S I_{L L}, S I_{V P}, S I_{V S I}=$ Severity indices of post-contingent line loading, voltage profile and voltage stability indices respectively.

\section{Computation of Network Composite Overall Severity Index} (NCOSI)

The network composite overall severity index (NCOSI) is obtained by adding the three overall severity indices as shown in Fig.4.

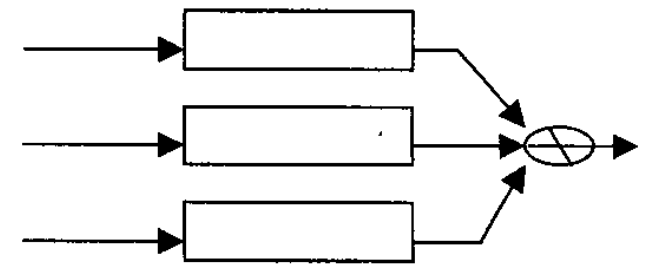

Fig. 4. Paralle] operated fuzzy Inference Systems (FIS) 


\section{TENCON $2003 / 758$}

\section{SELECTION OF SUITABLE LOCATIONS OF UPFC UNDER}

\section{NETWORK CONTINGENCY}

It is proposed to improve the performance of the system by selecting suitable locations for UPFC using all of its benefits under network contingencies. For a given contingency the possible locations of UPFC are analyzed. A contingency may involve a line having UPFC, and thus two locations for UPFC are selected based on the best performance of the system. These two UPFCs may take care of many network contingencies.

\section{UPFC equivalent circuit}

The UPFC equivalent circuit for steady state model is shown in Fig.5.

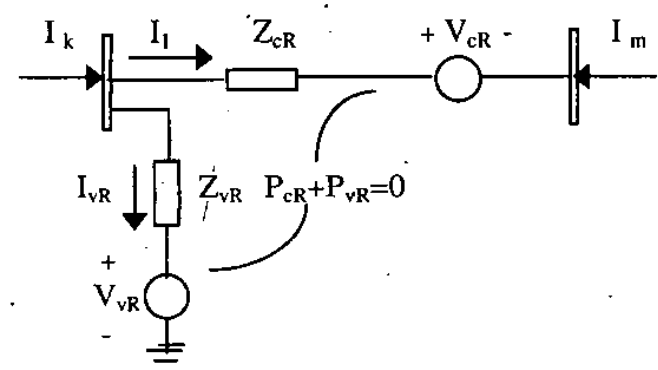

Fig.5. UPFC equivalent circuit

The equivalent circuit consists of two ideal voltage sources and are:

$$
\begin{aligned}
& V_{c R}=V_{c R}\left(\cos \theta_{c R}+\sin \theta_{c R}\right) \\
& V_{\nu R}=V_{\nu R}\left(\cos \theta_{\nu R}+\sin \theta_{\nu R}\right)
\end{aligned}
$$

Where $V_{v R}$ and $\theta_{v R}$ are the controllable magnitude $\left(V_{v R \text { min }} \leq V_{v R} \leq V_{v R \text { max }}\right)$ and angle $\left(0 \leq \theta_{v R} \leq 2 \pi\right)$ of the shunt voltage source .The magnitude $V_{c R}$ and angle $\theta_{c R}$ of the series voltage source are controlled between limits $\quad\left(V_{c R \text { min }} \leq V_{c R} \leq V_{c R \max }\right) \quad$ and angle $\left(0 \leq \theta_{c R} \leq 2 \pi\right)$ respectively [11].

\section{Approach for UPFC location}

The following are the major steps involved in the approach for selection of UPFC location under a given network contingency.

Step 1: Identify the transmission corridors for the given network.

Step 2: Select some transmission lines as suitable locations for each transmission corridor.

Step 3: Perform the power flow/voltage stability analysis with UPFC connected in selected line for each transmission corridor for a given network contingency.

Step 4: Compute performance of voltage stability improvement indices $\mathscr{L}_{\mathrm{L}}$ of the load buses.

$$
\mathscr{L}_{\mathrm{L}}=\sum_{j=g+1}^{n}\left(L_{j}^{\text {new }}-L_{j}^{\text {old }}\right)
$$

Where

$L_{j}^{\text {old }}=$ Initial voltage stability index of a load bus $j$ under a contingency

$L_{j}^{\text {new }}=$ New voltage stability index of a load bus $\mathrm{j}$

The negative value of $\mathscr{L}_{\mathrm{L}}$ indicates that the overall system stability/security is improved.

The positive value of $\mathscr{L}_{\mathrm{L}}$ indicates that the overall system stability/security is not improved.

Step 5: Prepare a list indicating location of UPFC, the value of $\mathscr{L}_{\mathrm{L}}$ and maximum value of voltage Stability Index $L_{\max }$.

Step 6: From the above list we can identify the most suitable location for UPFC, which gives maximum value for $\mathscr{L}_{\mathrm{L}}$.

\section{A REAL LIFE 36-BUS EQUIVALENT EHV SYSTEM}

\section{STUDIES}

\section{Contingency Ranking}

The fuzzy approach for contingency ranking is applied on a real life system of 36-bus equivalent EHV power system network shown in Fig6.The system has 9 generator buses and 27 other buses. The load is represented at 27 numbers of buses. The system total peak load is about 4657MW; 1789MVAR. There are shunt reactors connected at various $400 \mathrm{kV}$ buses for transient over-voltage protection. The ranking of all the line outage contingency cases using the fuzzy approach is shown in Table 2 . The proposed method of contingency ranking is able to distinguish clearly the actual severity of the system considering line loading, voltage profiles and voltage stability indices from one contingency to other. Hence the fuzzy method eliminates problem of masking effect of other methods for network contingency ranking.

\section{UPFC Location}

Since most of the contingencies may not pose threat the system security/stability those of contingencies that pose serious system security/stability problems are selected. For the 36-bus system rank 1,2 and 3 network contingencies are considered for illustrative purpose of the approach. 


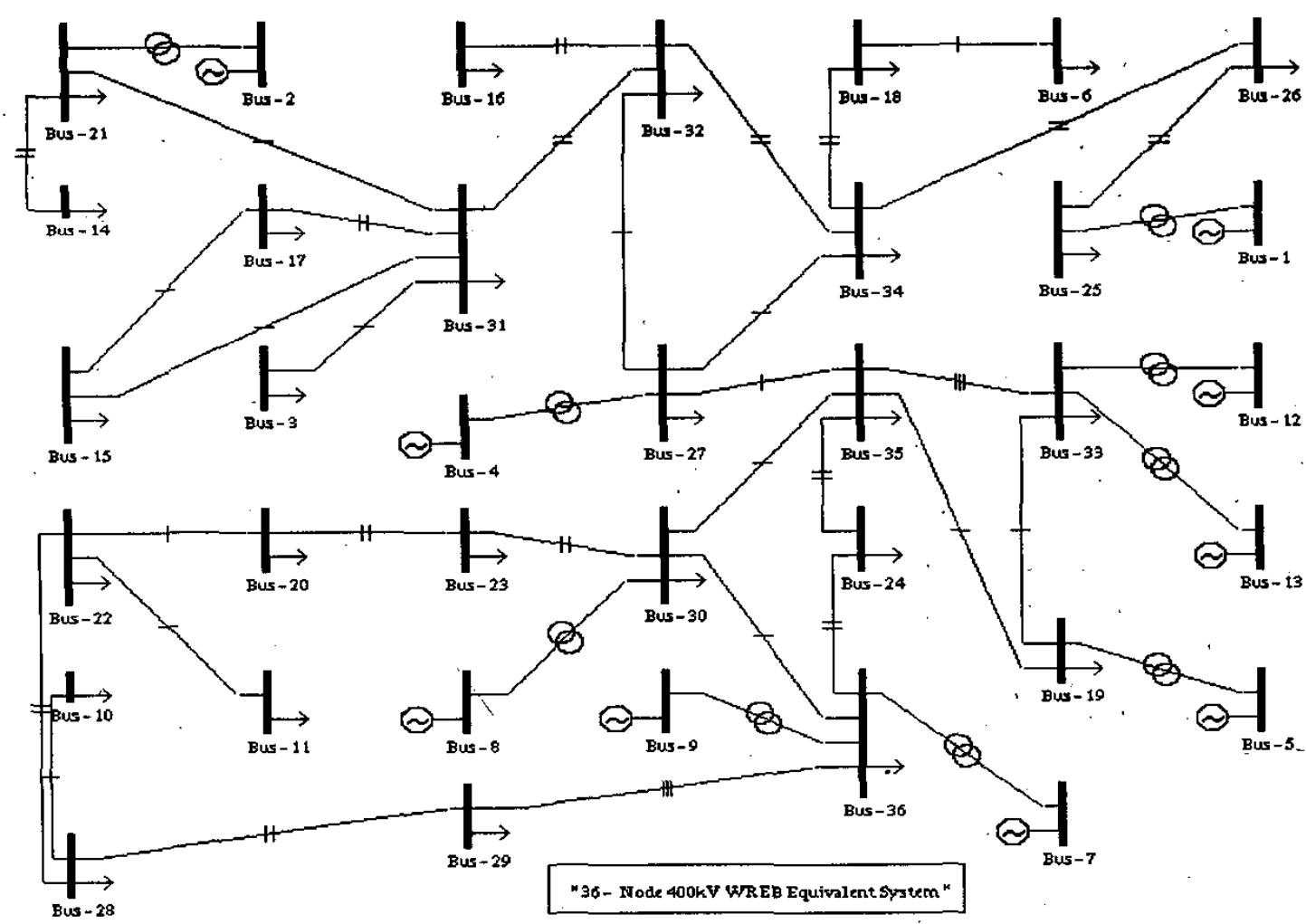

Fig.6. A Real life 36-node Equivalent EHV System

Table 2

Overall Severity Indices and Ranks of the corresponding line outages

\begin{tabular}{|c|c|c|c|c|c|}
\hline Line & OSI & & & & \\
& & & & & \\
\hline OSI & OSI $_{\mathrm{VSI}}$ & NCOSI & Rank \\
\hline $14-21$ & 890 & 1.438 & 772 & 3100 & 16 \\
\hline $17-15$ & 934 & 1.4058 & 892 & 3230 & 10 \\
\hline $18-34$ & 896 & 1.374 & 814 & 3084 & 19 \\
\hline $19-33$ & 877 & 1.580 & 757 & 3215 & 11 \\
\hline $22-28$ & 858 & 1.520 & 885 & 3262 & 8 \\
\hline $25-26$ & 1224 & 1.180 & 947 & 3350 & 5 \\
\hline $24-35$ & 869 & 1.341 & 803 & 3013 & 21 \\
\hline $26-34$ & 1089 & 1.187 & 886 & 3161 & 14 \\
\hline $27-35$ & 1148 & 1.205 & 919 & 3271 & 7 \\
\hline $27-34$ & 1038 & 1.546 & 993 & 3577 & 2 \\
\hline $30-27$ & 1021 & 1.725 & 848 & 3594 & 1 \\
\hline $30-35$ & 1041 & 1.202 & 870 & 3112 & 15 \\
\hline $31-17$ & 895 & 1.362 & 835 & 3092 & 17 \\
\hline $31-32$ & 991 & 1.294 & 880 & 3166 & 13 \\
\hline $32-16$ & 877 & 1.365 & 798 & 3041 & 20 \\
\hline $32-34$ & 1088 & 1.211 & 880 & 3178 & 12 \\
\hline $33-25$ & 1224 & 1.491 & 813 & 3527 & 3 \\
\hline $35-19$ & 896 & 1.398 & 795 & 3089 & 18 \\
\hline $35-33$ & 1033 & 1.428 & 794 & 3255 & 9 \\
\hline $36-24$ & 905 & 1.647 & 746 & 3297 & 6 \\
\hline $36-30$ & 951 & 1.299 & 891 & 3427 & 4 \\
\hline
\end{tabular}

Rank-1 Contingency (line 34-27 outage)
In this contingency for a peak load condition the overall total real power loss is $246.78(5.01 \%)$. The minimum voltage $V_{\min }$ is 0.907 p.u at bus 13 and the maximum voltage stability index $L_{\max }$ is 0.874 at bus 10 . ' The summary of results of UPFC placement under rank -1 contingency is given in Table 3 .

Table 3

Summary of results of UPFC placement under rank-1 contingency

\begin{tabular}{|c|c|c|c|}
\hline $\begin{array}{c}U P F C \\
\text { Line }\end{array}$ & $\mathscr{L}_{L} \ldots$ & $L_{\max }$ & $V_{\min }$ \\
\hline $36-30$ & -0.1362 & 0.8578 & 0.9143 \\
\hline $35-30$ & 0.7111 & 0.8324 & 0.9186 \\
\hline $35-27$ & 1.3548 & 0.8582 & 0.9389 \\
\hline
\end{tabular}

From the above Table 3 it can be observed that the value of $\mathscr{L}_{\mathrm{L}}$ is negative for the UPFC location in the line $36-30$ which indicates the improvement in system stability/security and is positive for other UPFC locations which indices the no improvement of system stability/security .The values of $L_{\max }$ and $V_{\min }$ are also better from initial values under contingency. Therefore the suitable line for UPFC placement is 36-30 under the rank-1 contingency.

Rank-2 Contingency (line 30-27 outage) 


\section{TENCON $2003 / 760$}

In this contingency for a peak load condition the overall total real power loss is $242.3 \mathrm{MW}(4.95 \%)$. The minimum voltage $V_{\min }$ is 0.897 p.u at bus 16 and the maximum voltage stability index $L_{\max }$ is 0.856 at bus 10 . The summary of results of UPFC placement under rank -2 contingency is given in Table 4 .

Table 4

Summary of results of UPFC placement under rank-2 contingency

\begin{tabular}{|l|c|c|c|}
\hline $\begin{array}{l}U P F C \\
\text { Line }\end{array}$ & $\mathscr{L}_{L}$ & $L_{\max }$ & $V_{\min }$ \\
\hline $36-30$ & -1.8824 & 0.7169 & 0.9681 \\
\hline $35-30$ & -1.0231 & 0.7797 & 0.9618 \\
\hline $35-27$ & 0.2443 & 0.8625 & 0.9537 \\
\hline
\end{tabular}

It can be observed from the above Table 4 that the value of $\mathscr{L}_{\mathrm{L}}$ is negative for the UPFC location in the lines $36-30$ and $35-30$, which indicates the improvement in system stability/security and is positive for the UPFC location in the line 35-27 which indicates no improvement of system stability/security. Since the value of $\mathscr{L}_{\mathrm{L}}$ is more negative for the UPFC location in line 36-30 compared to the UPFC location in the line 35-30. Therefore the most suitable line for UPFC placement is $36-30$ under the rank-2 contingency.

\section{Rank-3 Contingency (line 33-25 outage)}

In this contingency for a peak load condition the overall total real power loss is $233.92 \mathrm{MW}(4.78 \%)$. The minimum voltage $V_{\min }$ is $0.956 \mathrm{p} . \mathrm{u}$ at bus 10 and the maximum voltage stability index $L_{\max }$ is 0.909 at bus 10 . The summary of results of UPFC placement under rank -3 contingency is given in Table 5 .

Table 5

Summary of results of UPFC placement under rank-3 contingency

\begin{tabular}{|c|c|c|c|}
\hline $\begin{array}{c}U P F C \\
\text { Line }\end{array}$ & $\mathscr{L}_{L}$ & $L_{\max }$ & $V_{\min }$ \\
\hline $36-30$ & -1.9396 & 0.7169 & 0.9668 \\
\hline $35-30$ & -1.0435 & 0.7797 & 0.9770 \\
\hline $35-27$ & 0.0681 & 0.8625 & 0.9360 \\
\hline
\end{tabular}

It can be observed from the above Table 5 that the value of $\mathscr{L}_{\mathrm{L}}$ is negative for the UPFC location in the lines $36-30$ and $35-30$, which indicates the improvement in system stability/security and is positive for the UPFC location in the line 35-27 which indicates no improvement of system stability/security. Since the value of $\mathscr{L}_{\mathrm{L}}$ is more negative for the UPFC location in line $36-30$ compared to the UPFC location in the line 35-30. Therefore the most suitable line for UPFC placement is $36-30$ under the rank-3 contingency.

It can be concluded that, from all the above contingency cases, the most suitable location for all the above contingencies is the line 36-30 which can take care of all the contingencies and next is the line 35-30.

\section{Conclusions}

In this paper, in addition to real power loadings and bus voltages, the voltage stability indices at the load buses are also used as the post-contingent quantities to evaluate the network composite contingency ranking. The fuzzy contingency ranking method eliminates the masking effect. The selection of UPFC location under contingencies uses the voltage stability L-index improvements of load buses. A set of most severe contingencies, in the order of severity, is identified which needs additional supporting devices. Based on the above set of network contingencies, a few transmission corridors are considered for placement of UPFC devices. For each contingency, analyses are carried out with placement of UPFC in different transmission corridors. The proposed approach for UPFC location has been tested under simulated conditions on a few sample power systems and the results for a 36-node real-life equivalent EHV power network are presented for illustration purposes.

\section{REFERENCES}

F.D. Galiana, Bound estimates of the severity of line outages IEEE Transactions on PAS 1984, Vol.103, 2612-2624.

T.S. Sidhu, L.Cui, Contingency screening for steady state security analysis by using FFT and ANNs, IEEE Transactions on Power Systems 2000; Vol.15; 421-426.

G.C. Ejebe, B.F.Wollenberg, Automatic contingency selection, - IEEE Transactions on PAS 1974, Vol.93, 859-869.

T.F. Halpin, R.Fischl, R.Fink, Analysis of automatic contingency selection algorithms, IEEE Transactions on PAS 1984, Vol.103, 938-945.

Yuan Y.H, Kuo H.C. Fuzzy Set Based Contingency Ranking, IEEE Transactions on Power Systems 1992, 7(3), 1189-1196.

Bansilal, Thukaram D, Parthasarathy K. Optimal reactive powe dispatch algorithm for voltage stability improvement, Electrical Power and Energy Systems 1996, 18(7), 461-468.

Udupa A N, Thukaram D, Parthasarathy K. An expert fuzzy control approach to voltage stability enhancement, Electrical Power and Energy Systems 1999, 21, 279-287.

IEEE Power Engineering Society/CIGRE: FACTS overview, IEEE service center, Piscataway, N.J., 1995. Special Issue.95TP108.

IEEE Power Engineering Society/CIGRE: FACTS applications, IEEE service center, Piscataway, N.J., 1996. Special Issue.96TP116-0.

G.D. Galiana , et al., Assessment and control of the impact of FACTS devices on power system performance, IEEE Trans. on Power Systems., 1996,11(4), 1931-1936.

C.R Fuerte-Esquivel,E.Acha, Unified power flow controller: crifical comparison of Newton-Raphson UPFC algorithms in powerflowstudies,IEEProc.-ener.Tranm.Distrib.,144,(.5), 1997. 